\title{
Palladium-Catalyzed $\alpha$-Arylation of Dimethyl Malonate and Ethyl Cyanoacetate with o-Alkoxybromobenzenes for the Synthesis of Phenylacetic Acid, Esters and Phenylacetonitriles
}

\author{
José F. Cívicos ${ }^{a}$ \\ Paulo R. R. Costa*a \\ Jorge L. O. Domingos*b \\ a Instituto de Pesquisas de Produtos Naturais, Centro de \\ Ciências da Saúde, Universidade Federal do Rio de Janeiro, \\ 21941-590, Rio de Janeiro, RJ, Brazil \\ prrcosta2011@gmail.com \\ b Dep. Química Orgânica, Instituto de Química, Centro de \\ Tecnologia e Ciências, Universidade do Estado do Rio de \\ Janeiro, 20550-900, Rio de Janeiro, RJ, Brazil
}

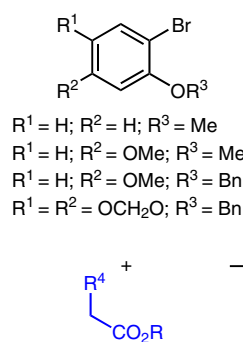

$\mathrm{R}=\mathrm{Me} ; \mathrm{R}^{4}=\mathrm{CO}_{2} \mathrm{Me}$ $\mathrm{R}=\mathrm{Et} ; \mathrm{R}^{4}=\mathrm{CN}$

Received: 28.07.2017

Accepted after revision: 01.08.2017

Published online: 22.08 .2017

DOI: 10.1055/s-0036-1588550; Art ID: so-2017-d0023-op

License terms: cc)

Abstract $\alpha$-Aryl malonate and $\alpha$-aryl cyano acetate moieties are found in the structures of many bioactive compounds. They are also key intermediates for the synthesis of many compounds such as isoflavonoids. In this work, we synthesized these compounds, with different patterns of substitution, starting with the palladium-catalyzed reaction of o-alkoxybromoarenes with dimethyl malonate or ethyl cyanoacetate. Under the conditions applied, moderate to good yields of arylmalonate monoesters, phenylacetic esters or acids, and benzylnitrile derivatives were obtained.

Key words $\alpha$-aryl malonates, $\alpha$-aryl- $\alpha$-cyanoacetates, $\alpha$-arylation reactions, palladium catalysis, decarboxylation

$\alpha$-Aryl malonates are found as substructures in bioactive compounds such as isoquinoline-1,3-dione, an HIV-1 integrase inhibitor, ${ }^{1}$ and in barbiturates such as alphenal. ${ }^{2}$ They can also be used as intermediates for the synthesis of 3-hydroxy-2-phenylpropanoic acid derivatives, through the reduction of one carboxylate group, to obtain tropic acid derivatives such as the tropane alkaloid scopolamine, an antiemetic drug. ${ }^{3}$ The decarboxylation reaction of $\alpha$-aryl dicarbonyl compounds leads to $\alpha$-phenylacetic acids, which are found for example in the structure of ibuprofen (antiinflammatory activity), ${ }^{4}$ in UPF523 (active in the CNS), ${ }^{5}$ and in JSTX-3 (a neurotoxin found in spider venom). ${ }^{6}$ They have also been used in the total synthesis of isoflavonoids such as cajanol and daidzein. ${ }^{7}$ Phenylacetonitriles are used in the synthesis of isoflavonoids, ${ }^{8}$ and reduction of the nitrile

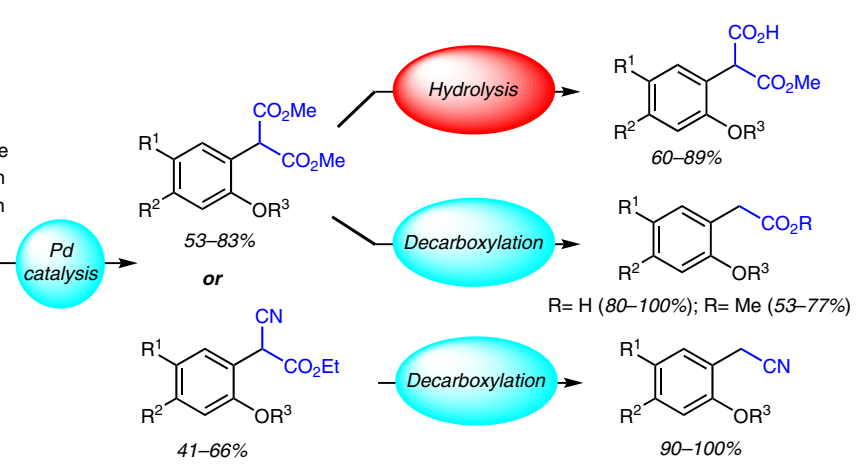

group gives rise to phenylethylamines. ${ }^{9}$ These are found in pharmaceuticals such as the antiarrithimic verapamil and the anticancer drug anastrazole. ${ }^{10}$ The structures of these compounds are shown in Figure 1.<smiles>O=C(NCc1ccc(F)cc1)C1C(=O)N(O)C(=O)c2cc([N+](=O)[O-])ccc21</smiles>

2-hydroxyisoquinoline$1,3(2 \mathrm{H}, 4 \mathrm{H})$-diones<smiles>CC(C)Cc1ccc(C(C)C(=O)O)cc1</smiles><smiles>O=Cc1ccc2c(c1)CCN2O</smiles><smiles>C=CC(C=C)(c1ccccc1)C1C(=O)NC(=O)NC1=O</smiles><smiles>CN1[C@@H]2CO[C@@H]1CC(OC(=O)[C@H](CO)c1ccccc1)C2</smiles>

scopolamine<smiles>COc1cc(O)c2c(c1)OCC(c1ccc(O)cc1OC)C2=O</smiles>
UPF-523<smiles>O=c1c(-c2ccc(O)cc2)coc2cc(O)ccc12</smiles>

daidzein<smiles>CCCCNC(=O)CCNCCCCCNC(=O)C(CO)NC(=O)Cc1ccc(O)cc1O</smiles><smiles>CCN(CCCC(C)(C)c1ccc(OC)c(OC)c1)CCCC(C)(C)N(C)CCc1ccc(OC)c(OC)c1</smiles>

Figure 1 Bioactive compounds with $\alpha$-arylmalonate, phenylacetic and benzylnitrile moieties 
The alkylation of malonates is a useful approach to prepare intermediates in organic synthesis. ${ }^{11}$ However, this approach is limited to primary and secondary alkyl halides and halobenzenes substituted with electron-withdrawing groups. ${ }^{12}$ Our interest lies in the synthesis of oxygenated $\alpha$ aryl carbonyl structures, which are used as precursors of isoflavonoid natural products, and these compounds cannot be prepared through the $\mathrm{S}_{\mathrm{N}} \mathrm{Ar}$ approach. ${ }^{12}$ In this paper, we report the synthesis of $\alpha$-arylmalonates $\left(\mathbf{1} ; \mathrm{R}^{1}=\mathrm{R}^{2}=\right.$ alkyl) and $\alpha$-arylcyanoacetates $\mathbf{5}$ by $\alpha$-arylation of malonates and cyanoacetates with aryl halides and their transformations into the desired compounds 2-6, as shown in Figure 2.<smiles>CC(=O)C(C(C)=O)c1ccc(O)cc1O</smiles><smiles>CC(=O)C(C(=O)O)c1ccc(O)cc1O</smiles><smiles>CC(=O)Cc1ccc(O)cc1O</smiles><smiles>O=C(O)Cc1ccc(O)cc1O</smiles>
4<smiles>CCOC(=O)c1ccc(O)cc1O</smiles>
5<smiles>N#CCc1ccc(O)cc1O</smiles>

6

Figure $2 \alpha$-Arylmalonates, phenylacetic acid derivatives, and phenylacetonitriles synthesized in this work

The $\alpha$-arylation reaction of carbonyl compounds has been studied since the early eighties, and more extensively and independently studied by Buchwald, Hartwig and Miura. ${ }^{13}$ Since then, various metal catalysts, ligands, and conditions have been developed for this reaction. ${ }^{14}$ Our approach was based on the $\alpha$-arylation of malonates and cyanoacetates catalyzed by palladium. Although these reactions have been well studied, little attention has been given to the use of the more sterically hindered $o$-alkoxy-bromoarenes as the arylating agents, leading to compounds of type $\mathbf{1}$ and $\mathbf{5}$. In addition, very few oxygenation patterns at the aromatic ring have been examined using these $\alpha$-arylations.

The $\alpha$-arylation step was optimized using the reaction of $o$-bromoanisole and dimethyl malonate or ethyl cyanoacetate (Scheme 1). After $20 \mathrm{~h}$ at $70^{\circ} \mathrm{C}$ in toluene in the presence of $\operatorname{Pd}_{2}(\mathrm{dba})_{3}$, the corresponding arylmalonate $\mathbf{1 a}$ was obtained in $60 \%$ yield. ${ }^{15}$ Aryl-cyano acetate 5a was prepared, under the same conditions $(15 \mathrm{~h})$, in $66 \%$ yield (Scheme 1). ${ }^{16}$ Both compounds were purified by flash column chromatography. Other $O$-protected bromoarenes were used under the same conditions and the $\alpha$-arylmalonates $\mathbf{1 b}-\mathbf{e}$ and $\alpha$-arylcyanoacetates $\mathbf{5 b}, \mathbf{c}$ were obtained in yields ranging from 41 to $83 \%$ (Scheme 1). By using this methodology, we were able to prepare derivatives that were 0 -benzylated in the ortho-position (41-83\%), which is a protecting group that can be removed under less drastic conditions than those used to remove methyl groups. ${ }^{17}$ This is the first time that compounds 1c, $\mathbf{1 d}$ and $\mathbf{5 c}$ have been synthesized.
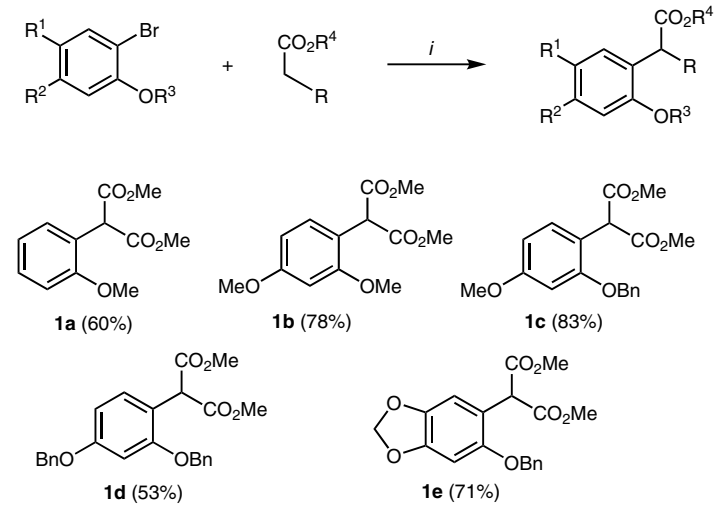<smiles>CCOC(=O)C(C#N)c1ccccc1OC</smiles>

$5 a(66 \%)$<smiles>CCOC(=O)C(C#N)c1ccc(OC)cc1OC</smiles>

5 b $(55 \%)$<smiles>CCOC(=O)C(C#N)c1ccc(OC)cc1OCC</smiles>

$5 c(41 \%)$
Scheme $1 \alpha$-Arylation reactions. Reagents and conditions: (i) $\mathrm{Pd}_{2}(\mathrm{dba})_{3}$ (2.5\% mol), $t \mathrm{Bu}_{3} \mathrm{PHBF}_{4}(10 \% \mathrm{~mol}), \mathrm{K}_{3} \mathrm{PO}_{4}$ (3 equiv), toluene, $20 \mathrm{~h} / 70{ }^{\circ} \mathrm{C}$ (for $\mathbf{1 a - e )} ; \mathrm{Na}_{3} \mathrm{PO}_{4}$ (3 equiv), $15 \mathrm{~h} / 70{ }^{\circ} \mathrm{C}$ (for $\mathbf{5 a - c}$ ).

The monohydrolysis of arylmalonates has been described under basic or acidic conditions. ${ }^{18}$ In our hands, in the presence of $\mathrm{KOH}$ in $\mathrm{THF}-\mathrm{H}_{2} \mathrm{O}$ at $0{ }^{\circ} \mathrm{C}$ the arylmonomethyl malonate $\mathbf{2 a}$ was obtained in low yield as a mixture with the unreacted arylmalonate and the decarboxylation product. The same outcome was observed when $\mathrm{KOH} / \mathrm{MeOH}$ at $35^{\circ} \mathrm{C}$ was used. However, the desired product 2a was obtained in good yield when $\mathrm{KOH}$ was used in a mixture of $\mathrm{MeOH} / \mathrm{H}_{2} \mathrm{O}(10: 1, \mathrm{v} / \mathrm{v})$, at $36{ }^{\circ} \mathrm{C}$ after $2 \mathrm{~h}$ (Scheme 2 ). Under the same conditions, arylmalonates $\mathbf{1 b}$-e were hydrolyzed to the corresponding aryl monomethyl malonates $\mathbf{2 b}-\mathbf{e}$ in yields ranging from 70 to $89 \%$.
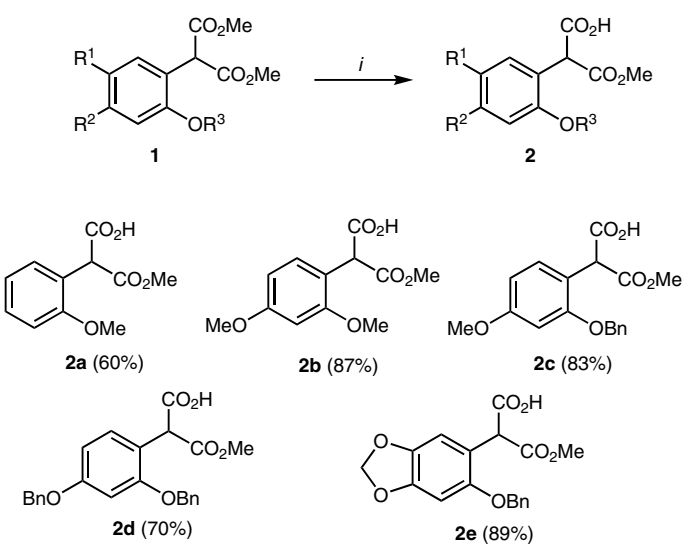

Scheme 2 Monohydrolysis of arylmalonates. Reagents and conditions: (i) $\mathrm{KOH}$ (2 equiv), $\mathrm{MeOH} / \mathrm{H}_{2} \mathrm{O}(10: 1, \mathrm{v} / \mathrm{v}), 36^{\circ} \mathrm{C}, 2 \mathrm{~h}$.

The one-pot hydrolysis followed by decarboxylation was successfully used to obtain, selectively, either arylacetate esters or the arylacetic acids, by changing the reaction 
conditions. When the temperature was increased to $80^{\circ} \mathrm{C}$, methyl esters of arylacetates 3a-e were obtained in moderate to high yields (Scheme 3). By increasing the amount of base from 2 to 3 equivalents and raising the temperature to $90{ }^{\circ} \mathrm{C}$, under microwave irradiation, arylacetic acids $4 \mathbf{a}-\mathbf{e}$ were produced in high yields (80-100\%) (Scheme 3).

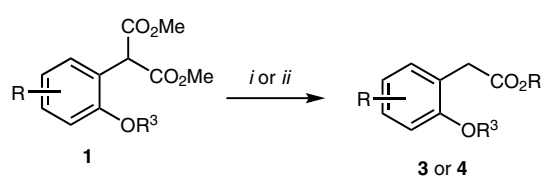<smiles>CCCC(=O)Cc1ccccc1OC</smiles>

3a $\mathrm{R}^{1}=\mathrm{Me}(75 \%)$ 4a $R^{1}=H(80 \%)$<smiles>COc1ccc(CC(=O)O)c(OC)c1</smiles><smiles>CCCC(=O)Cc1ccc(OC)cc1OCC</smiles>

$3 c \mathrm{R}^{1}=\mathrm{Me}(76 \%)$ 4c $\mathrm{R}^{1}=\mathrm{H}(84 \%)$<smiles>CCCOc1cc(OCc2ccccc2)ccc1CC(=O)CCC</smiles><smiles>O=C(O)COc1cc2c(cc1CC(=O)O)OCO2</smiles>

3d $\mathrm{R}^{1}=\mathrm{Me}(53 \%)$ 4d $R^{1}=H(100 \%)$ 3e $\mathrm{R}^{1}=\mathrm{Me}(70 \%)$ $4 e R^{1}=H(86 \%)$
Scheme 3 Hydrolysis/decarboxylation reactions. Reagents and conditions: For 3a-e: (i) $\mathrm{KOH}$ (2 equiv), $\mathrm{MeOH} / \mathrm{H}_{2} \mathrm{O}(10: 1, \mathrm{v} / \mathrm{v}), 90^{\circ} \mathrm{C}, 2.5 \mathrm{~h}$; for 4a-e: (ii) $\mathrm{KOH}$ (3 equiv), $\mathrm{MeOH} / \mathrm{H}_{2} \mathrm{O}(10: 1, \mathrm{v} / \mathrm{v}), 90^{\circ} \mathrm{C}, 150 \mathrm{~W}, 20$ min.

The hydrolysis/decarboxylation of aryl-cyanoacetates $\mathbf{5}$ was performed under the same conditions and benzylnitriles $\mathbf{6 a}-\mathbf{b}$ were obtained in high yields (Scheme 4).

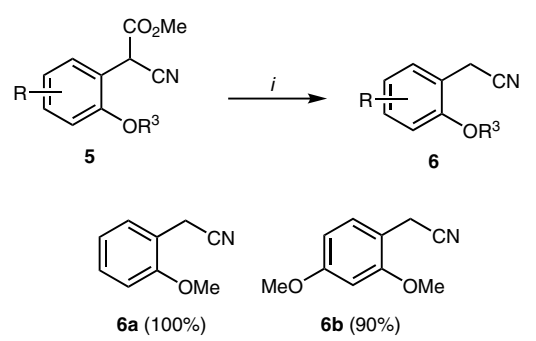

Scheme 4 Hydrolysis/decarboxylation reactions. Reagents and conditions: (i) $\mathrm{KOH}$ ( 3 equiv), $\mathrm{MeOH} / \mathrm{H}_{2} \mathrm{O}(10: 1, \mathrm{v} / \mathrm{v}), 90^{\circ} \mathrm{C}, 150 \mathrm{~W}, 20 \mathrm{~min}$.

In conclusion, selected products from palladiumcatalyzed $\alpha$-arylation reactions of dimethyl malonates and ethyl cyanoacetates with bromoarenes were obtained in moderate to good yields. Some of these compounds, with the benzyl protecting group on the phenol functionality (1c, 1d, 5c), ${ }^{19,20}$ were obtained for the first time in this work.

The arylmalonates could be transformed directly into the phenylacetic acids or the corresponding methyl esters (3a-e, 4a-e). Arylacetonitriles $\mathbf{6 a - b}$ could also be prepared from arylcyanoacetates $\mathbf{5 a - c}$ through one-pot hydrolysis followed by decarboxylation.
${ }^{1} \mathrm{H}$ NMR $(400 \mathrm{MHz})$ and ${ }^{13} \mathrm{C}$ NMR $(75 \mathrm{MHz})$ spectra were obtained with a Varian Gemini-200 with $\mathrm{CDCl}_{3}$ as solvent and TMS as internal standard unless otherwise stated. High-resolution mass spectra were obtained at $70 \mathrm{eV}$ by electron impact with direct insertion on a Micromass MM12F. Analytical TLC was performed on Merck aluminum sheets with silica gel $60 \mathrm{~F}_{254}$. For flash chromatography, Merck silica gel $60(0.040-0.063 \mathrm{~mm})$ was used. Melting points were determined with a Fisatom 430 apparatus and are uncorrected.

\section{Arylation of Dimethyl Malonate with Aryl Bromides; General Pro- cedure}

Dimethyl malonate $(1 \mathrm{mmol})$, aryl bromide $(1.1 \mathrm{mmol})$, $\left[\mathrm{HP}(\mathrm{tBu})_{3}\right] \mathrm{BF}_{4}$ $(0.10 \mathrm{mmol}), \mathrm{Pd}_{2}(\mathrm{dba})_{3}(0.025 \mathrm{mmol})$, and $\mathrm{K}_{3} \mathrm{PO}_{4}(3.0 \mathrm{mmol})$ were placed in a $25 \mathrm{~mL}$ round-bottomed flask with a magnetic stirrer. The flask was closed with a septum, the contents were placed under an argon atmosphere and anhydrous toluene $(5.0 \mathrm{~mL})$ was added. The heterogeneous mixture was stirred at $70{ }^{\circ} \mathrm{C}$ and the reaction was monitored by TLC. Upon complete consumption of the aryl bromide, the crude reaction mixture was filtered through a plug of Celite ${ }^{\circledR}$, extracted with EtOAc $(3 \times 10 \mathrm{~mL})$, washed with brine, dried with anhydrous $\mathrm{Na}_{2} \mathrm{SO}_{4}$, filtered, and concentrated in vacuo. The residue was purified by chromatography on silica gel (1:9, EtOAc/hexanes).

\section{Dimethyl 2-(2-Methoxyphenyl)malonate (1a) $)^{21}$}

Yield: $0.143 \mathrm{~g}$ (60\%); yellow oil; $R_{f} 0.16$ (1:9, EtOAc/hexanes).

${ }^{1} \mathrm{H}$ NMR $\left(\mathrm{CDCl}_{3}, 400 \mathrm{MHz}\right): \delta=7.36-7.28(\mathrm{~m}, 2 \mathrm{H}), 6.97(\mathrm{td}, J=7.5$, $1.0 \mathrm{~Hz}, 1 \mathrm{H}), 6.90(\mathrm{~d}, J=8.1 \mathrm{~Hz}, 1 \mathrm{H}), 5.16(\mathrm{~s}, 1 \mathrm{H}), 3.82(\mathrm{~s}, 3 \mathrm{H}), 3.75(\mathrm{~s}$, $6 \mathrm{H})$.

${ }^{13} \mathrm{C}$ NMR $\left(\mathrm{CDCl}_{3}, 100 \mathrm{MHz}\right): \delta=169.0,156.8,129.5,129.4,121.5$, $120.7,110.7,55.6,52.7,50.7$.

\section{Dimethyl 2-(2,4-Dimethoxyphenyl)malonate (1b)}

Yield: $0.209 \mathrm{~g}$ (78\%); brown solid; $\mathrm{mp} 60-62{ }^{\circ} \mathrm{C} ; R_{f} 0.26$ (1:9 EtOAc/hexanes).

${ }^{1} \mathrm{H} \mathrm{NMR}\left(\mathrm{CDCl}_{3}, 400 \mathrm{MHz}\right): \delta=7.25(\mathrm{~d}, J=8.5 \mathrm{~Hz}, 1 \mathrm{H}), 6.50$ (dd, $J=8.5$, $2.4 \mathrm{~Hz}, 1 \mathrm{H}), 6.46(\mathrm{~d}, J=2.3 \mathrm{~Hz}, 1 \mathrm{H}), 5.07(\mathrm{~s}, 1 \mathrm{H}), 3.79(\mathrm{~s}, 6 \mathrm{H}), 3.74(\mathrm{~s}$, $6 \mathrm{H})$.

${ }^{13} \mathrm{C}$ NMR $\left(\mathrm{CDCl}_{3}, 100 \mathrm{MHz}\right): \delta=169.3,168.9,160.8,157.8,130.1$, 113.9, 104.5, 98.6, 55.6, 55.3, 52.7, 50.1, 48.9.

HRMS: $m / z$ calcd for $\mathrm{C}_{13} \mathrm{H}_{16} \mathrm{O}_{6}$ : 268.0947; found: 268.0946 .

\section{Dimethyl 2-(2-(Benzyloxy)-4-methoxyphenyl)malonate (1c)}

Yield: $0.285 \mathrm{~g}$ (83\%); yellow solid; $\mathrm{mp} 61-63^{\circ} \mathrm{C} ; R_{f} 0.21$ (1:9 EtOAc/hexanes).

${ }^{1} \mathrm{H} \mathrm{NMR}\left(\mathrm{CDCl}_{3}, 400 \mathrm{MHz}\right): \delta=7.40-7.32(\mathrm{~m}, 4 \mathrm{H}), 7.30(\mathrm{dd}, J=6.0$, $2.7 \mathrm{~Hz}, 1 \mathrm{H}), 7.25(\mathrm{~d}, J=8.1 \mathrm{~Hz}, 1 \mathrm{H}), 6.51(\mathrm{~s}, 1 \mathrm{H}), 6.49(\mathrm{~d}, J=2.4 \mathrm{~Hz}$, $1 \mathrm{H}), 5.12(\mathrm{~s}, 1 \mathrm{H}), 5.02(\mathrm{~s}, 2 \mathrm{H}), 3.73(\mathrm{~s}, 3 \mathrm{H}), 3.68(\mathrm{~s}, 6 \mathrm{H})$.

${ }^{13} \mathrm{C}$ NMR $\left(\mathrm{CDCl}_{3}, 100 \mathrm{MHz}\right): \delta=169.3,160.8,157.0,136.6,130.2$, 128.64, 128.0, 127.3, 114.5, 105.0, 99.9, 70.4, 55.5, 52.8, 50.7.

HRMS: $m / z$ calcd for $\mathrm{C}_{19} \mathrm{H}_{20} \mathrm{O}_{6}$ : 344.1260 ; found: 344.1259 .

\section{Dimethyl 2-(2,4-Bis(benzyloxy)phenyl)malonate (1d)}

Yield: $0.223 \mathrm{~g}$ (53\%); yellow solid; mp $103-105^{\circ} \mathrm{C} ; R_{f} 0.16(1: 9$ EtOAc/hexanes).

${ }^{1} \mathrm{H} \mathrm{NMR}\left(\mathrm{CDCl}_{3}, 400 \mathrm{MHz}\right): \delta=7.42-7.29(\mathrm{~m}, 8 \mathrm{H}), 7.24(\mathrm{q}, J=4.5 \mathrm{~Hz}$, $2 \mathrm{H}), 7.14(\mathrm{~d}, J=8.1 \mathrm{~Hz}, 1 \mathrm{H}), 6.60(\mathrm{~d}, J=2.2 \mathrm{~Hz}, 1 \mathrm{H}), 6.58(\mathrm{dd}, J=8.4$, $2.3 \mathrm{~Hz}, 1 \mathrm{H}), 5.12(\mathrm{~s}, 1 \mathrm{H}), 5.03(\mathrm{~s}, 2 \mathrm{H}), 5.01(\mathrm{~s}, 2 \mathrm{H}), 3.71(\mathrm{~s}, 6 \mathrm{H})$. 
${ }^{13} \mathrm{C}$ NMR $\left(\mathrm{CDCl}_{3}, 100 \mathrm{MHz}\right): \delta=169.4,160.1,157.1,139.5,136.8$, 136.6, 128.8, 128.7, 128.2, 128.1, 127.8, 127.7, 127.3, 123.0, 106.0, $100.8,70.4,70.3,52.83,52.83,50.8$.

HRMS: $m / z$ calcd for $\mathrm{C}_{25} \mathrm{H}_{24} \mathrm{O}_{6}$ : 420.1573; found: 420.1572 .

Dimethyl 2-(6-(Benzyloxy)benzo[d][1,3]dioxol-5-yl)malonate (1e) Yield: $0.254 \mathrm{~g}$ (71\%); brown solid; $\mathrm{mp} 87-89^{\circ} \mathrm{C} ; R_{f} 0.10$ (1:9 EtOAc/hexanes).

${ }^{1} \mathrm{H}$ NMR $\left(\mathrm{CDCl}_{3}, 400 \mathrm{MHz}\right): \delta=7.39-7.29(\mathrm{~m}, 5 \mathrm{H}), 6.88(\mathrm{~s}, 1 \mathrm{H}), 6.57$ (s, $1 \mathrm{H}), 5.90(\mathrm{~s}, 2 \mathrm{H}), 5.16(\mathrm{~s}, 1 \mathrm{H}), 5.00(\mathrm{~s}, 2 \mathrm{H}), 3.71(\mathrm{~s}, 6 \mathrm{H})$.

${ }^{13} \mathrm{C}$ NMR $\left(\mathrm{CDCl}_{3}, 125 \mathrm{MHz}\right): \delta=169.0,151.3,148.1,141.7,136.6$, 128.5, 128.1, 127.4, 127.2, 114.1, 109.2, 109.2, 101.4, 96.4, 96.3, 71.7, $52.8,52.7,50.7,50.5$.

HRMS: $m / z$ calcd for $\mathrm{C}_{19} \mathrm{H}_{18} \mathrm{O}_{7}$ : 358.1053; found: 358.1052 .

\section{Arylation of Ethyl Cyanoacetate with Aryl Bromides; General Pro- cedure}

Ethyl cyanoacetate $(1.1 \mathrm{mmol})$, aryl bromide $(1.0 \mathrm{mmol})$, $\left[\mathrm{HP}(\mathrm{tBu})_{3}\right] \mathrm{BF}_{4}(0.10 \mathrm{mmol}), \mathrm{Pd}_{2}(\mathrm{dba})_{3}\left(0.025 \mathrm{mmol}\right.$, and $\mathrm{Na}_{3} \mathrm{PO}_{4}(3.0$ $\mathrm{mmol}$ ) were placed in a $25 \mathrm{~mL}$ round-bottom flask. The flask was closed with a septum, the contents were placed under an argon atmosphere and anhydrous toluene $(5.0 \mathrm{~mL})$ was added. The heterogeneous reaction mixture was stirred at $70{ }^{\circ} \mathrm{C}$ and monitored by TLC. Upon complete consumption of the aryl bromide, the crude reaction mixture was filtered through a plug of Celite ${ }^{\circledR}$, extracted with EtOAc $(3 \times 10 \mathrm{~mL})$, washed with brine, dried with anhydrous $\mathrm{Na}_{2} \mathrm{SO}_{4}$, filtered, and concentrated in vacuo. The residue was purified by chromatography on silica gel (1:9 EtOAc/hexanes).

\section{Ethyl 2-Cyano-2-(2-methoxyphenyl)acetate (5a) $)^{22}$}

Yield: $0.144 \mathrm{~g}$ (66\%); yellow oil; $R_{f} 0.12$ (1:9 EtOAc/hexanes).

${ }^{1} \mathrm{H} \mathrm{NMR}\left(\mathrm{CDCl}_{3}, 400 \mathrm{MHz}\right): \delta=7.36(\mathrm{dd}, J=12.2,4.5 \mathrm{~Hz}, 2 \mathrm{H}), 6.99(\mathrm{t}$, $J=7.5 \mathrm{~Hz}, 1 \mathrm{H}), 6.93(\mathrm{~d}, J=8.2 \mathrm{~Hz}, 1 \mathrm{H}), 5.03(\mathrm{~s}, 1 \mathrm{H}), 4.24(\mathrm{dd}, J=7.2$, $4.2 \mathrm{~Hz}, 2 \mathrm{H}), 3.84(\mathrm{~s}, 3 \mathrm{H}), 1.27(\mathrm{t}, J=7.1 \mathrm{~Hz}, 2 \mathrm{H})$.

${ }^{13} \mathrm{C}$ NMR $\left(\mathrm{CDCl}_{3}, 125 \mathrm{MHz}\right): \delta=165.1,156.4,130.6,129.3,120.9$, 119.0, 115.8, 111.0, 62.8, 55.6, 38.1, 24.5, 13.8 .

\section{Ethyl 2-Cyano-2-(2,4-dimethoxyphenyl)acetate (5b) $)^{23}$}

Yield: $0.137 \mathrm{~g}$ (55\%); red brown oil; $R_{f} 0.13$ (1:9 EtOAc/hexanes).

${ }^{1} \mathrm{H}$ NMR $\left(\mathrm{CDCl}_{3}, 400 \mathrm{MHz}\right): \delta=7.39$ (ddd, $J=12.3,6.6,1.4 \mathrm{~Hz}, 2 \mathrm{H}$ ), $7.01(\mathrm{td}, J=7.6,0.9 \mathrm{~Hz}, 1 \mathrm{H}), 6.94(\mathrm{~d}, J=8.2 \mathrm{~Hz}, 1 \mathrm{H}), 5.03(\mathrm{~s}, 1 \mathrm{H}), 4.27$ (qd, $J=7.1,1.4 \mathrm{~Hz}, 2 \mathrm{H}), 3.87$ (s, $3 \mathrm{H}), 1.32-1.27(\mathrm{~m}, 3 \mathrm{H})$.

${ }^{13} \mathrm{C}$ NMR $\left(\mathrm{CDCl}_{3}, 125 \mathrm{MHz}\right): \delta=165.5,161.7,157.5,130.0,116.2$, $111.5,104.9,98.9,62.9,55.7,55.5,37.6,14.0$.

\section{Ethyl 2-(2-(Benzyloxy)-4-methoxyphenyl)-2-cyanoacetate (5c)}

Yield: $0.139 \mathrm{~g}$ ( $41 \%$ ); yellow oil; $R_{f} 0.14$ (1:9 EtOAc/hexanes).

${ }^{1} \mathrm{H} \mathrm{NMR}\left(\mathrm{CDCl}_{3}, 500 \mathrm{MHz}\right): \delta=7.44-7.37(\mathrm{~m}, 4 \mathrm{H}), 7.33$ (dd, $J=13.5$, $7.8 \mathrm{~Hz}, 2 \mathrm{H}), 6.54(\mathrm{~d}, J=6.9 \mathrm{~Hz}, 2 \mathrm{H}), 5.08(\mathrm{q}, J=11.5 \mathrm{~Hz}, 2 \mathrm{H}), 4.95(\mathrm{~s}$, $1 \mathrm{H}), 4.15(\mathrm{q}, J=7.0 \mathrm{~Hz}, 2 \mathrm{H}), 3.80(\mathrm{~s}, 3 \mathrm{H}), 1.19(\mathrm{t}, J=7.1 \mathrm{~Hz}, 3 \mathrm{H})$.

${ }^{13} \mathrm{C}$ NMR $\left(\mathrm{CDCl}_{3}, 125 \mathrm{MHz}\right): \delta=165.5,161.7,156.6,136.1,130.3$, $128.8,128.8,128.7,128.7,128.2,127.4,116.2,111.8,105.2,100.1$, 70.5, 62.9, 55.5, 38.0, 13.9 .

HRMS: $m / z$ calcd for $\mathrm{C}_{19} \mathrm{H}_{19} \mathrm{NO}_{4}$ : 325.1314 ; found: 325.1314 .

\section{Selective Monohydrolysis of Dimethyl Arylmalonates; General Procedure}

Dimethyl arylmalonate $(1.0 \mathrm{mmol})$ and $\mathrm{KOH}(2.0 \mathrm{mmol})$, followed by $\mathrm{MeOH}(1.0 \mathrm{~mL})$ and $\mathrm{H}_{2} \mathrm{O}(0.1 \mathrm{~mL})$ were placed in a $25 \mathrm{~mL}$ roundbottom flask with a magnetic stirrer. The mixture was stirred at $36{ }^{\circ} \mathrm{C}$ for $2 \mathrm{~h}$, then the reaction was quenched by the addition of water $(5$ $\mathrm{mL})$. Unreacted ester was removed by EtOAc extraction $(2 \times 5 \mathrm{~mL})$ and the carboxylic acid was obtained by acidification of the aqueous phase to $\mathrm{pH} 2$ with $10 \% \mathrm{HCl}$, extracted with EtOAc $(3 \times 5 \mathrm{~mL})$, washed with brine, dried over anhydrous $\mathrm{Na}_{2} \mathrm{SO}_{4}$, filtered, and concentrated on a rotary evaporator.

3-Methoxy-2-(2-methoxyphenyl)-3-oxopropanoic Acid (2a)

Yield: $0.134 \mathrm{~g}(60 \%)$; brown oil.

${ }^{1} \mathrm{H} \mathrm{NMR}\left(\mathrm{CDCl}_{3}, 500 \mathrm{MHz}\right): \delta=7.22(\mathrm{~d}, J=7.5 \mathrm{~Hz}, 1 \mathrm{H}), 7.20-7.15(\mathrm{~m}$, $1 \mathrm{H}), 6.81(\mathrm{t}, J=7.4 \mathrm{~Hz}, 1 \mathrm{H}), 6.77(\mathrm{~d}, J=8.2 \mathrm{~Hz}, 1 \mathrm{H}), 6.40(\mathrm{~s}, 1 \mathrm{H}), 4.90$ (s, $1 \mathrm{H}), 3.67(\mathrm{~s}, 3 \mathrm{H}), 3.57$ (s, $3 \mathrm{H})$.

${ }^{13} \mathrm{C} \mathrm{NMR}\left(\mathrm{CDCl}_{3}, 100 \mathrm{MHz}\right): \delta=173.1,169.6,156.8,129.8,129.8$, $121.4,120.8,110.9,55.7,53.0,50.9$.

HRMS: $m / z$ calcd for $\mathrm{C}_{11} \mathrm{H}_{12} \mathrm{O}_{5}$ : 224.0685; found: 224.0684 .

\section{2-(2,4-Dimethoxyphenyl)-3-methoxy-3-oxopropanoic Acid (2b) ${ }^{24}$} Yield: $0.221 \mathrm{~g}(87 \%)$; pale-brown solid; $\mathrm{mp} 108-110{ }^{\circ} \mathrm{C}$.

${ }^{1} \mathrm{H} \mathrm{NMR}\left(\mathrm{CDCl}_{3}, 500 \mathrm{MHz}\right): \delta=7.26(\mathrm{~d}, J=3.4 \mathrm{~Hz}, 1 \mathrm{H}), 6.51(\mathrm{dd}, J=8.5$, $2.4 \mathrm{~Hz}, 1 \mathrm{H}), 6.47(\mathrm{~d}, J=2.3 \mathrm{~Hz}, 1 \mathrm{H}), 4.92(\mathrm{~s}, 1 \mathrm{H}), 3.81(\mathrm{~s}, 3 \mathrm{H}), 3.80(\mathrm{~s}$, $3 \mathrm{H}), 3.76(\mathrm{~s}, 3 \mathrm{H})$.

${ }^{13} \mathrm{C}$ NMR $\left(\mathrm{CDCl}_{3}, 100 \mathrm{MHz}\right): \delta=173.8,169.7,160.9,157.8,130.4$, 113.6, 104.7, 98.7, 55.7, 55.4, 52.9, 50.3.

2-(2-(Benzyloxy)-4-methoxyphenyl)-3-methoxy-3-oxopropanoic Acid (2c)

Yield: $0.274 \mathrm{~g}$ (83\%); pale-brown solid; $\mathrm{mp} 165-167^{\circ} \mathrm{C}$.

${ }^{1} \mathrm{H} \mathrm{NMR}\left(\mathrm{CDCl}_{3}, 500 \mathrm{MHz}\right): \delta=8.98(\mathrm{~s}, 1 \mathrm{H}), 7.35(\mathrm{q}, J=7.9 \mathrm{~Hz}, 4 \mathrm{H})$, 7.31-7.23 (m, 2 H), 6.62-6.38 (m, 2 H), 5.04 (s, 1 H), 5.03 (s, 2 H), 3.76 (s, $3 \mathrm{H}), 3.69(\mathrm{~s}, 3 \mathrm{H})$.

${ }^{13} \mathrm{C}$ NMR $\left(\mathrm{CDCl}_{3}, 100 \mathrm{MHz}\right): \delta=171.5,171.0,161.1,156.9,136.3$, 131.0, 128.7, 128.2, 127.4, 114.4, 105.2, 100.2, 70.6, 55.5, 53.2, 50.7.

HRMS: $\mathrm{m} / z$ calcd for $\mathrm{C}_{18} \mathrm{H}_{18} \mathrm{O}_{6}: 330.1103$; found: 330.1103 .

\section{2-(2,4-Bis(benzyloxy)phenyl)-3-methoxy-3-oxopropanoic Acid} (2d)

Yield: $0.284 \mathrm{~g}$ (70\%); pale-brown solid; $\mathrm{mp} 90-92^{\circ} \mathrm{C}$.

${ }^{1} \mathrm{H}$ NMR $\left(\mathrm{CDCl}_{3}, 500 \mathrm{MHz}\right): \delta=7.50-7.24(\mathrm{~m}, 11 \mathrm{H}), 6.69-6.54(\mathrm{~m}$, $2 \mathrm{H}), 5.08-4.96(\mathrm{~m}, 4 \mathrm{H}), 4.87(\mathrm{~s}, 1 \mathrm{H}), 3.71(\mathrm{~s}, 3 \mathrm{H})$.

${ }^{13} \mathrm{C}$ NMR $\left(\mathrm{CDCl}_{3}, 125 \mathrm{MHz}\right): \delta=171.5,170.3,160.5,156.8,136.7$, 136.3, 128.8, 128.7, 128.3, 128.2, 128.2, 127.8, 127.7, 127.5, 127.4, $114.8,106.1,100.9,70.6,70.4,53.2,50.6$.

HRMS: $m / z$ calcd for $\mathrm{C}_{24} \mathrm{H}_{22} \mathrm{O}_{6}$ : 406.1416; found: 406.1416 .

\section{2-(6-(Benzyloxy)benzo[d][1,3]dioxol-5-yl)-3-methoxy-3-oxo-} propanoic Acid (2e)

Yield: $0.306 \mathrm{~g}$ (89\%); yellow oil.

${ }^{1} \mathrm{H} \mathrm{NMR}\left(\mathrm{CDCl}_{3}, 400 \mathrm{MHz}\right): \delta=8.51(\mathrm{~s}, 1 \mathrm{H}), 7.43-7.23(\mathrm{~m}, 5 \mathrm{H}), 6.88$ (s, $1 \mathrm{H}), 6.57$ (s, $1 \mathrm{H}), 5.91$ (s, $2 \mathrm{H}), 5.09$ (s, $1 \mathrm{H}), 5.00$ (s, $2 \mathrm{H}), 3.72$ (s, $3 \mathrm{H})$. 
${ }^{13} \mathrm{C}$ NMR $\left(\mathrm{CDCl}_{3}, 125 \mathrm{MHz}\right): \delta=172.5,170.1,151.4,148.5,141.8$, 136.5, 128.8, 128.74, 128.68, 127.5, 127.4, 113.8, 109.8, 101.7, 96.4, $71.8,53.1,50.8$.

HRMS: $m / z$ calcd for $\mathrm{C}_{18} \mathrm{H}_{16} \mathrm{O}_{7}$ : 344.0896 ; found: 344.0896 .

\section{Synthesis of $\alpha$-Arylacetates; General Procedure}

Dimethyl arylmalonate $(1.0 \mathrm{mmol})$ and $\mathrm{KOH}(2.0 \mathrm{mmol})$, followed by $\mathrm{MeOH}(1.0 \mathrm{~mL})$ and $\mathrm{H}_{2} \mathrm{O}(0.1 \mathrm{~mL})$ were placed in a $25 \mathrm{~mL}$ round-bottom flask with a magnetic stirrer. The mixture was stirred at $80^{\circ} \mathrm{C}$ for $2.5 \mathrm{~h}$, then the reaction was quenched by the addition of water $(5 \mathrm{~mL})$. The $\alpha$-arylacetate was removed by EtOAc extraction $(3 \times 5 \mathrm{~mL})$ and the carboxylic acid was obtained by acidification of the aqueous phase to $\mathrm{pH} 2$ with $10 \% \mathrm{HCl}$, extracted with EtOAc $(3 \times 5 \mathrm{~mL})$, washed with brine, dried over anhydrous $\mathrm{Na}_{2} \mathrm{SO}_{4}$, filtered, and concentrated on a rotary evaporator.

\section{Methyl 2-(2-Methoxyphenyl)acetate (3a) $)^{25}$}

Yield: $0.135 \mathrm{~g}(75 \%)$; yellow oil.

${ }^{1} \mathrm{H} \mathrm{NMR}\left(\mathrm{CDCl}_{3}, 400 \mathrm{MHz}\right): \delta=7.27-7.22(\mathrm{~m}, 1 \mathrm{H}), 7.16(\mathrm{~d}, J=7.4 \mathrm{~Hz}$, $1 \mathrm{H}), 6.90(\mathrm{t}, J=7.2 \mathrm{~Hz}, 1 \mathrm{H}), 6.86(\mathrm{~d}, J=8.2 \mathrm{~Hz}, 1 \mathrm{H}), 3.80(\mathrm{~s}, 3 \mathrm{H}), 3.67$ (s, $3 \mathrm{H}), 3.62(\mathrm{~s}, 2 \mathrm{H})$.

${ }^{13} \mathrm{C}$ NMR $\left(\mathrm{CDCl}_{3}, 100 \mathrm{MHz}\right): \delta=172.41,157.55,130.92,128.65$, $123.03,120.56,110.52,55.50,51.98,35.83$.

\section{Methyl 2-(2,4-Dimethoxyphenyl)acetate (3b $)^{26}$.}

Yield: $0.162 \mathrm{~g}$ (77\%); yellow oil.

${ }^{1} \mathrm{H} \mathrm{NMR}\left(\mathrm{CDCl}_{3}, 400 \mathrm{MHz}\right): \delta=7.08(\mathrm{~d}, J=8.6 \mathrm{~Hz}, 1 \mathrm{H}), 6.48-6.41(\mathrm{~m}$, $2 \mathrm{H}), 3.80$ (s, $3 \mathrm{H}), 3.79$ (s, $3 \mathrm{H}), 3.68(\mathrm{~s}, 3 \mathrm{H}), 3.56(\mathrm{~s}, 2 \mathrm{H})$.

${ }^{13} \mathrm{C}$ NMR $\left(\mathrm{CDCl}_{3}, 125 \mathrm{MHz}\right): \delta=172.7,160.3,158.5,131.2,115.5$, 104.1, 98.6, 55.5, 55.4, 51.9, 35.1 .

\section{Methyl 2-(2-(Benzyloxy)-4-methoxyphenyl)acetate (3c) $)^{27}$}

Yield: $0.217 \mathrm{~g}(76 \%)$; brown oil.

${ }^{1} \mathrm{H} \mathrm{NMR}\left(\mathrm{CDCl}_{3}, 400 \mathrm{MHz}\right): \delta=7.45-7.28(\mathrm{~m}, 5 \mathrm{H}), 7.11(\mathrm{~d}, J=8.2 \mathrm{~Hz}$, $1 \mathrm{H}), 6.52(\mathrm{~d}, J=2.2 \mathrm{~Hz}, 1 \mathrm{H}), 6.47$ (dd, $J=8.2,2.3 \mathrm{~Hz}, 1 \mathrm{H}), 5.05(\mathrm{~s}$, $2 \mathrm{H}), 3.78$ (s, $3 \mathrm{H}), 3.63(\mathrm{~s}, 3 \mathrm{H}), 3.61(\mathrm{~s}, 2 \mathrm{H})$.

${ }^{13} \mathrm{C}$ NMR $\left(\mathrm{CDCl}_{3}, 100 \mathrm{MHz}\right): \delta=172.75,160.22,157.54,137.00$, 131.36, 128.61, 127.93, 127.19, 115.92, 104.54, 99.91, 70.04, 55.50, $51.95,35.55$.

\section{Methyl 2-(2,4-Bis(benzyloxy)phenyl)acetate (3d) ${ }^{28}$}

Yield: $0192 \mathrm{~g}$ (53\%); yellow solid; $\mathrm{mp} 78-80{ }^{\circ} \mathrm{C}$ (lit. 74.5-75 ${ }^{\circ} \mathrm{C}$ ).

${ }^{1} \mathrm{H} \mathrm{NMR}\left(\mathrm{CDCl}_{3}, 400 \mathrm{MHz}\right): \delta=7.44-7.28(\mathrm{~m}, 10 \mathrm{H}), 7.10(\mathrm{~d}, J=8.3 \mathrm{~Hz}$, $1 \mathrm{H}), 6.60(\mathrm{~d}, J=2.2 \mathrm{~Hz}, 1 \mathrm{H}), 6.54(\mathrm{dd}, J=8.2,2.3 \mathrm{~Hz}, 1 \mathrm{H}), 5.02(\mathrm{~d}, J=$ $4.0 \mathrm{~Hz}, 4 \mathrm{H}), 3.62(\mathrm{~s}, 3 \mathrm{H}), 3.61(\mathrm{~s}, 2 \mathrm{H})$.

${ }^{13} \mathrm{C} \mathrm{NMR}\left(\mathrm{CDCl}_{3}, 100 \mathrm{MHz}\right): \delta=172.7,159.4,157.5,136.9,136.9$, 131.3, 128.7, 128.5, 128.1, 127.9, 127.6, 127.1, 116.1, 105.5, 100.6, $70.2,69.9,51.9,35.5$

\section{Methyl 2-(6-(Benzyloxy)benzo[d][1,3]dioxol-5-yl)acetate (3e)}

Yield: $0.210 \mathrm{~g}$ (70\%); white solid; $\mathrm{mp} 105-107^{\circ} \mathrm{C}$.

${ }^{1} \mathrm{H} \mathrm{NMR}\left(\mathrm{CDCl}_{3}, 500 \mathrm{MHz}\right): \delta=7.47-7.23(\mathrm{~m}, 5 \mathrm{H}), 6.70(\mathrm{~s}, 1 \mathrm{H}), 6.56$ (s, $1 \mathrm{H}), 5.89$ (s, $2 \mathrm{H}), 4.99$ (s, $2 \mathrm{H}), 3.63$ (s, $3 \mathrm{H}$ ), 3.58 (s, $2 \mathrm{H}$ ).

${ }^{13} \mathrm{C} \mathrm{NMR}\left(\mathrm{CDCl}_{3}, 100 \mathrm{MHz}\right): \delta=172.5,151.6,147.4,141.3,137.0$, 128.6, 128.0, 127.2, 115.6, 110.7, 101.3, 96.3, 71.3, 52.0, 35.8.

HRMS: $\mathrm{m} / \mathrm{z}$ calcd for $\mathrm{C}_{17} \mathrm{H}_{16} \mathrm{O}_{5}$ : 300.0998 ; found: 300.0997 .

\section{Synthesis of $\alpha$-Arylacetic Acids; General Procedure}

A $10-\mathrm{mL}$ MW vessel was charged with dimethyl arylmalonate $(0.20$ $\mathrm{mmol})$ and $\mathrm{KOH}(0.60 \mathrm{mmol})$, followed by $\mathrm{MeOH}(2.0 \mathrm{~mL})$ and $\mathrm{H}_{2} \mathrm{O}$ $(0.2 \mathrm{~mL})$. The vessel was sealed with a pressure lock, and the mixture was heated under microwave irradiation $(150 \mathrm{~W})$ at $90{ }^{\circ} \mathrm{C}$ for $20 \mathrm{~min}$ in a CEM Discover MW reactor. After cooling to r.t., the reaction mixture was extracted with EtOAc $(3 \times 10 \mathrm{~mL})$, and the $\alpha$-arylacetic acid was obtained by acidification of the aqueous phase to $\mathrm{pH} 2$ with $10 \%$ $\mathrm{HCl}$, extracted with EtOAc $(3 \times 5 \mathrm{~mL})$, washed with brine, dried over anhydrous $\mathrm{Na}_{2} \mathrm{SO}_{4}$, filtered, and concentrated on a rotary evaporator.

\section{2-(2-Methoxyphenyl)acetic Acid (4a) ${ }^{29}$}

Yield: $0.026 \mathrm{~g}(80 \%)$; pale-brown solid; $\mathrm{mp} 115-117^{\circ} \mathrm{C}$ (lit. 119 $\left.121^{\circ} \mathrm{C}\right)$.

${ }^{1} \mathrm{H} \mathrm{NMR}\left(\mathrm{CDCl}_{3}, 500 \mathrm{MHz}\right): \delta=7.29-7.24(\mathrm{~m}, 1 \mathrm{H}), 7.18(\mathrm{~d}, J=7.3 \mathrm{~Hz}$, $1 \mathrm{H}), 6.92(\mathrm{t}, J=7.4 \mathrm{~Hz}, 1 \mathrm{H}), 6.88(\mathrm{~d}, J=8.2 \mathrm{~Hz}, 1 \mathrm{H}), 3.82(\mathrm{~s}, 3 \mathrm{H}), 3.66$ (s, $2 \mathrm{H})$.

${ }^{13} \mathrm{C}$ NMR $\left(\mathrm{CDCl}_{3}, 100 \mathrm{MHz}\right): \delta=178.2,157.6,131.1,129.0,122.4$, 120.7, 110.6, 55.6, 35.9.

\section{2-(2,4-Dimethoxyphenyl)acetic Acid (4b) $)^{30}$}

Yield: $0.034 \mathrm{~g}$ (87\%); white solid; $\mathrm{mp} 90-92{ }^{\circ} \mathrm{C}$ (lit. 99-102 ${ }^{\circ} \mathrm{C}$ ). ${ }^{1} \mathrm{H} \mathrm{NMR}\left(\mathrm{CDCl}_{3}, 400 \mathrm{MHz}\right): \delta=7.08(\mathrm{~d}, J=7.8 \mathrm{~Hz}, 1 \mathrm{H}), 6.51-6.40(\mathrm{~m}$, $2 \mathrm{H}), 3.80$ (s, $6 \mathrm{H}), 3.59$ (s, $2 \mathrm{H})$.

${ }^{13} \mathrm{C}$ NMR $\left(\mathrm{CDCl}_{3}, 100 \mathrm{MHz}\right): \delta=177.9,160.5,158.5,131.4,114.9$, $104.3,98.8,55.6,55.5,35.2$.

\section{2-(2-(Benzyloxy)-4-methoxyphenyl)acetic Acid (4c) ${ }^{31}$}

Yield: $0.046 \mathrm{~g}(84 \%)$; pale-yellow solid; $\mathrm{mp} 115-117^{\circ} \mathrm{C}$.

${ }^{1} \mathrm{H} \mathrm{NMR}\left(\mathrm{CDCl}_{3}, 500 \mathrm{MHz}\right): \delta=7.41-7.28(\mathrm{~m}, 5 \mathrm{H}), 7.11(\mathrm{~d}, J=8.3 \mathrm{~Hz}$, $1 \mathrm{H}), 6.52(\mathrm{~d}, J=2.0 \mathrm{~Hz}, 1 \mathrm{H}), 6.48(\mathrm{dd}, J=8.2,2.2 \mathrm{~Hz}, 1 \mathrm{H}), 5.05$ (s, $2 \mathrm{H}), 3.78$ (s, $3 \mathrm{H}), 3.64$ (s, $2 \mathrm{H})$.

${ }^{13} \mathrm{C}$ NMR $\left(\mathrm{CDCl}_{3}, 125 \mathrm{MHz}\right): \delta=177.7,160.3,157.5,136.9,131.5$, 128.7, 128.6, 127.9, 127.1, 127.1, 115.7, 104.6, 99.9, 70.1, 55.5, 35.7.

\section{2-(2,4-Bis(benzyloxy)phenyl)acetic Acid (4d) ${ }^{32}$}

Yield: $0.070 \mathrm{~g}$ (100\%); pale-brown solid; mp $134-136{ }^{\circ} \mathrm{C}$ (lit. $137-$ $\left.137.5^{\circ} \mathrm{C}\right)$.

${ }^{1} \mathrm{H}$ NMR $\left(\mathrm{CDCl}_{3}, 400 \mathrm{MHz}\right): \delta=7.28-7.04(\mathrm{~m}, 1 \mathrm{H}), 6.92(\mathrm{~d}, J=7.2 \mathrm{~Hz}$, $1 \mathrm{H}), 6.39(\mathrm{~s}, 1 \mathrm{H}), 6.28(\mathrm{~d}, J=7.4 \mathrm{~Hz}, 1 \mathrm{H}), 4.77(\mathrm{~s}, 1 \mathrm{H}), 4.71(\mathrm{~s}, 1 \mathrm{H})$, $3.38(\mathrm{~s}, 1 \mathrm{H})$.

${ }^{13} \mathrm{C}$ NMR $\left(\mathrm{CDCl}_{3}, 100 \mathrm{MHz}\right): \delta=177.4,158.6,157.2,137.1,131.4$, 128.6, 128.5, 127.9, 127.8, 127.7, 127.2, 118.9, 105.7, 101.2, 70.1, 69.9, 29.8 .

\section{2-(6-(Benzyloxy)benzo[d][1,3]dioxol-5-yl)acetic acid (4e) $)^{33}$}

Yield: $0.049 \mathrm{~g}(86 \%)$; brown solid; $\mathrm{mp} 119-122^{\circ} \mathrm{C}$.

${ }^{1} \mathrm{H}$ NMR $\left(400 \mathrm{MHz}\right.$, acetone- $\left.d_{6}\right): \delta=7.42(\mathrm{~d}, J=7.4 \mathrm{~Hz}, 2 \mathrm{H}), 7.34(\mathrm{t}, J=$ $7.4 \mathrm{~Hz}, 2 \mathrm{H}), 7.29$ (d, J = 7.3 Hz, $1 \mathrm{H}), 6.71(\mathrm{~s}, 1 \mathrm{H}), 6.66(\mathrm{~s}, 1 \mathrm{H}), 5.86$ (s, $2 \mathrm{H}), 5.00$ (s, $2 \mathrm{H}), 3.54$ (s, $2 \mathrm{H})$.

${ }^{13} \mathrm{C}$ NMR $\left(\mathrm{CDCl}_{3}, 125 \mathrm{MHz}\right): \delta=172.9,152.6,148.0,142.0,138.5$, $129.5,128.9,128.8,128.2,127.9,117.2,111.5,111.4,102.0,97.1,71.7$, 35.7.

\section{Synthesis of $\alpha$-Arylacetonitriles; General Procedure}

A $10-\mathrm{mL}$ MW vessel was charged with ethyl arylcyanoacetate $(0.20$ $\mathrm{mmol})$ and $\mathrm{KOH}(0.60 \mathrm{mmol})$, followed by $\mathrm{MeOH}(2.0 \mathrm{~mL})$ and $\mathrm{H}_{2} \mathrm{O}$ $(0.2 \mathrm{~mL})$. The vessel was sealed with a pressure lock, and the mixture 
was heated under microwave irradiation $(150 \mathrm{~W})$ at $90{ }^{\circ} \mathrm{C}$ for $20 \mathrm{~min}$ in a CEM Discover MW reactor. After cooling to r.t., the reaction mixture was extracted with EtOAc $(3 \times 10 \mathrm{~mL})$, and the $\alpha$-arylacetonitrile was obtained by acidification of the aqueous phase to $\mathrm{pH} 2$ with $10 \%$ $\mathrm{HCl}$, extracted with EtOAc $(3 \times 5 \mathrm{~mL})$, washed with brine, dried over anhydrous $\mathrm{Na}_{2} \mathrm{SO}_{4}$, filtered, and concentrated on a rotary evaporator.

\section{2-(2-Methoxyphenyl)acetonitrile (6a) ${ }^{34}$}

Yield: $0.029 \mathrm{~g}$ (100\%); yellow oil.

${ }^{1} \mathrm{H} \mathrm{NMR}\left(\mathrm{CDCl}_{3}, 400 \mathrm{MHz}\right): \delta=7.30(\mathrm{dd}, J=15.9,7.9 \mathrm{~Hz}, 2 \mathrm{H}), 6.94(\mathrm{t}$, $J=7.4 \mathrm{~Hz}, 1 \mathrm{H}), 6.86$ (d, J = 8.2 Hz, $1 \mathrm{H}), 3.82(\mathrm{~s}, 3 \mathrm{H}), 3.64(\mathrm{~s}, 2 \mathrm{H})$.

${ }^{13} \mathrm{C} \mathrm{NMR}\left(\mathrm{CDCl}_{3}, 100 \mathrm{MHz}\right): \delta=156.6,129.4,129.1,120.6,118.5$, $118.0,110.4,55.3,18.5$.

\section{2-(2,4-Dimethoxyphenyl)acetonitrile (6b) $)^{35}$}

Yield: $0.032 \mathrm{~g}$ (90\%); white solid; $\mathrm{mp} 70-72{ }^{\circ} \mathrm{C}$ (lit. $76{ }^{\circ} \mathrm{C}$ ).

${ }^{1} \mathrm{H} \mathrm{NMR}\left(\mathrm{CDCl}_{3}, 400 \mathrm{MHz}\right): \delta=7.23(\mathrm{~d}, J=8.0 \mathrm{~Hz}, 1 \mathrm{H}), 6.48(\mathrm{~d}, J=$ $8.4 \mathrm{~Hz}, 2 \mathrm{H}), 3.84(\mathrm{~s}, 2 \mathrm{H}), 3.81(\mathrm{~s}, 2 \mathrm{H}), 3.61(\mathrm{~s}, 2 \mathrm{H})$.

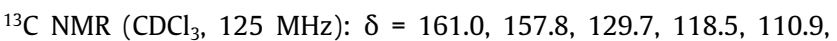
104.3, 98.7, 55.6, 55.5, 18.2.

\section{Funding Information}

We thank the Brazilian agencies Coordenação de Aperfeiçoamento de Pessoal de Nível Superior (CAPES; BJT-2014), Conselho Nacional de Desenvolvimento Científico e Tecnológico (CNPq), and Fundação Carlos Chagas Filho de Amparo à Pesquisa do Estado do Rio de Janeiro (FAPERJ).

\section{Supporting Information}

Supporting information for this article is available online at https://doi.org/10.1055/s-0036-1588550.

\section{References}

(1) Suchaud, V.; Bailly, F.; Lion, C.; Calmels, C.; Andréola, M. L.; Christ, F.; Debyser, Z.; Cotelle, P. J. Med. Chem. 2014, 57, 4640.

(2) Martin, J. R.; Godel, T.; Hunkeler, W.; Jenck, F.; Moreau, J.-L.; Sleight, A. J.; Widmer, U. Psychopharmacological Agents, In KirkOthmer Encyclopedia of Chemical Technology; 2000.

(3) Nocquet, P.-A.; Opatz, T. Eur. J. Org. Chem. 2016, 1156.

(4) Bogdan, A. R.; Poe, S. L.; Kubis, D. C.; Broadwater, S. J.; McQuade, D. T. Angew. Chem. Int. Ed. 2009, 48, 8547.

(5) Costantino, G.; Pellicciari, R. J. Med. Chem. 1996, 39, 3998.

(6) Barslund, A. F.; Poulsen, M. H.; Bach, T. B.; Lucas, S.; Kristensen, A. S.; Strømgaard, K. J. Nat. Prod. 2011, 74, 483.

(7) (a) Goel, A.; Kumar, A.; Hemberger, Y.; Raghuvanshi, A.; Jeet, R.; Tiwari, G.; Knauer, M.; Kureel, J.; Singh, A. K.; Gautam, A.; Trivedi, R.; Singh, D.; Bringmann, G. Org. Biomol. Chem. 2012, 10, 9583. (b) Basha, G. M.; Yadav, S. K.; Srinuvasarao, R.; Prasanthi, S.; Ramu, T.; Mangarao, N.; Siddaiah, V. Can. J. Chem. 2013, 91, 763.

(8) (a) Vasquez-Martinez, Y.; Ohri, R. V.; Kenyon, V.; Holman, T. R.; Sepúlveda-Boza, S. Bioorg. Med. Chem. 2007, 15, 7408. (b) Liu, J.; Yang, Z.; Luo, S.; Hao, Y.; Ren, J.; Su, Y.; Wang, W.; Li, R. Synth. Commun. 2014, 44, 3296.
(9) Delorme, D.; Gregor, V.; Roberts, E.; Sun, E. WO 9967203, 1999; Chem. Abstr. 1999, 132, 49802

(10) Fleming, F. F.; Yao, L.; Ravikumar, P. C.; Funk, L.; Shook, B. C. J. Med. Chem. 2010, 53, 7902.

(11) (a) Pearson, R. G. J. Am. Chem. Soc. 1949, 71, 2212. (b) Hoogenboom, B. E.; Ihrig, P. J.; Langsjoen, A. N.; Linn, C. J.; Mulder, S. D. J. Chem. Educ. 1991, 68, 689.

(12) (a) Yoshida, M.; Maeyama, Y.; Shishido, K. Tetrahedron 2012, 68, 9962. (b) Deng, P.; Lei, Y.; Zheng, X.; Li, S.; Wu, J.; Zhu, F.; Ong, B. S.; Zhang, Q. Dyes Pigm. 2016, 125, 407. (c) Yoshikawa, M.; Kamisaki, H.; Kunitomo, J.; Oki, H.; Kokubo, H.; Suzuki, A.; Ikemoto, T.; Nakashima, K.; Kamiguchi, N.; Harada, A.; Kimura, H.; Taniguchi, T. Bioorg. Med. Chem. 2015, 23, 7138.

(13) (a) Uno, M.; Seto, K.; Takahashi, S. J. Chem. Soc., Chem. Commun. 1984, 932. (b) Uno, M.; Seto, K.; Ueda, W.; Masuda, M.; Takahashi, S. Synthesis 1985, 506. (c) Sakamoto, T.; Kato, E.; Kondo, Y.; Yamanaka, H. Chem. Pharm. Bull. 1988, 36, 1664. (d) Palucki, M.; Buchwald, S. L. J. Am. Chem. Soc. 1997, 119, 11108. (e) Hartwig, J. F.; Hamann, B. C. J. Am. Chem. Soc. 1997, 119, 12382. (f) Satoh, T.; Kawamura, Y.; Miura, M.; Nomura, M. Angew. Chem. Int. Ed. Engl. 1997, 36, 1740.

(14) (a) Johansson, C. C. C.; Colacot, T. J. Angew. Chem. Int. Ed. 2010, 49, 676. (b) Bellina, F.; Rossi, R. Chem. Rev. 2010, 110, 1082.

(15) Beare, N. A.; Hartwig, J. F. J. Org. Chem. 2002, 67, 541.

(16) Stauffer, S. R.; Beare, N. A.; Stambuli, J. P.; Hartwig, J. F. J. Am. Chem. Soc. 2001, 123, 4641.

(17) Fernandes, T. A.; Domingos, J. L. O.; Rocha, L. I. A.; Medeiros, S.; Nájera, C.; Costa, P. R. R. Eur. J. Org. Chem. 2014, 1314.

(18) (a) Malki, F.; Touati, A.; Rahal, S.; Moulay, S. Asian J. Chem. 2011, 23, 961. (b) Niwayama, S.; Cho, H.; Lin, C. Tetrahedron Lett. 2008, 49, 4434. (c) Niwayama, S. J. Org. Chem. 2000, 65, 5834.

(19) Luo, M.; Liu, X.; Zu, Y.; Fu, Y.; Zhang, S.; Yao, L.; Efferth, T. Chem.Biol. Interact. 2010, 188, 151.

(20) Skouta, R.; Li, C. J. Tetrahedron Lett. 2007, 48, 8343.

(21) Baciocchi, E.; Dell'Aira, D.; Ruzziconi, R. Tetrahedron Lett. 1986, 27, 2763.

(22) Gandi, V. R.; Lu, Y. Chem. Commun. 2015, 16188.

(23) Daikin Kogyo Co. Ltd. Jpn. Kokai Tokkyo Koho JP 59051251 A 19840324, 1984; Chem Abstr. 1984, 101, 72461

(24) Kamijo, T.; Tsubaki, A.; Yamaguchi, T.; Hirata, K. Jpn. Kokai Tokkyo Koho JP 03024047 A 19910201, 1991; Chem Abstr. 1991, 115,49430

(25) Jaita, S.; Phakhodee, W.; Pattarawarapan, M. Synlett 2015, 26, 2006.

(26) Al-Maharik, N.; Botting, N. P. Tetrahedron 2004, 60, 1637.

(27) van Aardt, T. G.; van Rensburg, H.; Ferreira, D. Tetrahedron 2001, $57,7113$.

(28) Chiba, T.; Akizawa, T.; Matsukawa, M.; Nishi, M.; Kawai, N.; Yoshioka, M. Chem. Pharm. Bull. 1996, 44, 972.

(29) Morton, A. A.; Brachman, A. E. J. Am. Chem. Soc. 1954, 76, 2973.

(30) Bernier, D.; Brückner, R. Synthesis 2007, 2249.

(31) Gray, T. I.; Pelter, A.; Ward, R. S. Tetrahedron 1979, 35, 2539.

(32) Shih, T. L.; Ruiz-Sanchez, J.; Mrozik, H. Tetrahedron Lett. 1987, 28, 6015.

(33) Pinard, E.; Gaudry, M.; Hénot, F.; Thellend, A. Tetrahedron Lett. 1998, 39, 2739.

(34) Tseng, K.-N. T.; Rizzi, A. M.; Szymczak, N. K. J. Am. Chem. Soc. 2013, 135, 16352.

(35) Neill, K. G. J. Chem. Soc. 1953, 3454. 